\title{
Advances of Relativity Theory
}

\author{
Amrit Šorli Srečko \\ Bijective Physics Institute, Slovenia \\ sorli.bijective.physics@gmail.com
}

\begin{abstract}
In this article, a bijective research methodology is applied where every element in the model corresponds to exactly one element in physical reality. An element in the physical reality $X$ and the corresponding element in the model $Y$ are related by the bijective function. A bijective research methodology confirms that the Lorentz factor has its origin in the variable density of universal space. A lower value of the space density corresponds to the higher value of the Lorentz factor. Universal space is not "empty"; space is the primordial energy of the universe. Universal space is the absolute frame of reference for all observers as confirmed experimentally by the GPS system, which demonstrates that the relative rate of clocks is valid for all observers. A planet's perihelion precession and the Sagnac effect are the results of the space rotation.
\end{abstract}

Keywords: bijective research methodology, Lorentz factor, planet's precession, Sagnac effect, GPS, space dragging effect

PACS: 04.00

\section{Introduction}

Advances of Relativity Theory are based on three significant scientific discoveries:

1. Space-time is not a fundamental arena of the universe; time is not the 4th dimension of space. Time is merely the duration of motion in space [1].

2. Entanglement happens in space only and not in time. Space is the immediate medium of quantum entanglement [2].

3. Space is not "empty", space is the fundamental energy of the universe, in today physics called "superfluid quantum vacuum" [3].

GPS proves that the relative rate of clocks on satellites relative to the Earth's surface is valid for all observers, including aeroplanes, trains, ships, and cars [4, 5]. This experimental fact, along with everyday experience, suggests a revision to our understanding of the famous Gedankenexperiment of one observer at a train station and another observer on a passing train. Standard physics textbooks describe that a clock at the station runs faster for the observer on the train, and the clock on the train runs slower for the observer at the station. In classic relativity both observers have their own 'internal time' inside the reference system in which they exist and both have an 'external time' that exists in the other observer reference system. This interpretation features four distinct times: the proper time of the observer at the station, the proper time of the observer in the train, the external time of the observer at the 
station, and the external time of the observer on the train. On the other hand, GPS proves that the relative velocities of clocks at the station and on the train are equally related to the rate of clocks on orbiting satellites, so are valid for both observers. If this were not so, then GPS could not work properly.

This article is organized as follows. Section 2 presents the relation between the Einstein tensor and the variable density of universal space. Section 3 describes the origin of the Lorentz factor in the variable density of space. Analysis of the Special Relativity Theory is in Section 4. Section 5 shows the improvement of General Relativity Theory, gravity's impact on light from the perspective of variable density of the space and the origin of gravitational waves. Finally, Section 6 discusses the possibility for technological applications of variable density of universal space.

\section{The Einstein tensor and variable density of universal space}

Recent research developed a model of the variable density of a space where a given stellar object has a diminishing density of the space according to its mass, $m$ as

$$
m=\left(\rho_{\max }-\rho_{\min }\right) \cdot V
$$

where $\rho_{\min }$ is the density of the space on the surface of a given stellar object, $\rho_{\max }$ is the density of the space in interstellar space, and $V$ is the volume of the stellar object [6].

In General Relativity, the curvature of space is expressed with the Einstein tensor as

$$
\begin{gathered}
G_{\mu \nu}=\kappa \cdot T_{\mu \nu} \\
\text { with units of } \frac{1}{\mathrm{~m}^{2}}=\frac{\mathrm{m}}{\mathrm{kg}} \cdot \frac{\mathrm{kg}}{\mathrm{m}^{3}}
\end{gathered}
$$

where the curvature tensor $G_{\mu \nu}$ measures the curvature of space, $T_{\mu \nu}$ is the stress-energy tensor, and $\kappa=1,866 \cdot 10^{-26} \mathrm{mkg}^{-1}$ is the Einstein constant.

In this article, a heuristic formula is developed where a stress-energy tensor $T_{\mu \nu}$ is expressed by the difference of space density,

$$
G_{\mu \nu}=\kappa \cdot\left(\rho_{\max }-\rho_{\min }\right)
$$

where $\rho_{\min }$ is the density of the space on the surface of a given stellar object, and $\rho_{\max }$ is the density of the space in the interstellar space. Accordingly, Equation (3) will calculate the 
curvature tensor $G_{\mu \nu}$ for the surface of a black hole, on the Earth's surface, a neutron star's surface, and a proton's surface. As $\rho_{\max }$ is the same for all calculations, it will be left out.

The density of the space $\rho_{\min }$ on the surface of a black hole with the mass of the Sun and radius of 3000 metres is

$$
\begin{gathered}
\rho_{\min }=\rho_{\max }-1,759 \cdot 10^{19} \mathrm{~kg} / \mathrm{m3} \\
G_{\mu v}=1,866 \cdot 10^{-26} \mathrm{mkg}^{-1} *-1,759 \cdot 10^{19} \mathrm{kgm}^{-3} \\
G_{\mu v}=-3,28 \cdot 10^{-7} \mathrm{~m}^{-2}
\end{gathered}
$$

The density of the space $\rho_{\min }$ on the surface of a neutron star is

$$
\begin{gathered}
\rho_{\min }=\rho_{\max }-2.0 \cdot 10^{17} \mathrm{~kg} / \mathrm{m}^{3} \\
G_{\mu v}=1,866 \cdot 10^{-26} \mathrm{mkg}^{-1} *-2,0 \cdot 10^{17} \mathrm{kgm}^{-3} \\
G_{\mu \nu}=-3,73 \cdot 10^{-9} \mathrm{~m}^{-2}
\end{gathered}
$$

The density of the space $\rho_{\min }$ on the surface of the proton is

$$
\begin{gathered}
\rho_{\min }=\rho_{\max }-6.688 \cdot 10^{17} \mathrm{~kg} / \mathrm{m3} \\
G_{\mu v}=1,866 \cdot 10^{-26} \mathrm{mkg}^{-1} *-6,688 \cdot 10^{17} \mathrm{kgm}^{-3} \\
G_{\mu v}=-12,48 \cdot 10^{-9} \mathrm{~m}^{-2}
\end{gathered}
$$

The density of the space $\rho_{\min }$ on the surface of Earth is

$$
\begin{gathered}
\rho_{\min }=\rho_{\max }-5.514 \cdot 10^{3} \mathrm{~kg} / \mathrm{m}^{3} \\
G_{\mu v}=1,866 \cdot 10^{-26} \mathrm{mkg}^{-1} *-5,514 \cdot 10^{3} \mathrm{kgm}^{-3} \\
G_{\mu v}=-10,29 \cdot 10^{-23} \mathrm{~m}^{-2}
\end{gathered}
$$

Interstellar space: $\rho_{\min }=\rho_{\max }$

$$
G_{\mu v}=0 m^{-2}
$$


Black hole: $\quad \rho_{\min }=\rho_{\max }-1,759 \cdot 10^{19} \mathrm{kgm}^{-3} \quad G_{\mu \nu}=-3,28 \cdot 10^{-7} \mathrm{~m}^{-2}$

$\begin{array}{lll}\text { Neutron star: } & \rho_{\text {min }}=\rho_{\max }-2,0 \cdot 10^{17} \mathrm{kgm}^{-3} & G_{\mu \nu}=-3,73 \cdot 10^{-9} \mathrm{~m}^{-2} \\ \text { Proton: } & \rho_{\min }=\rho_{\max }-6.688 \cdot 10^{17} \mathrm{kgm}^{-3} & G_{\mu \nu}=-12,48 \cdot 10^{-9} \mathrm{~m}^{-2} \\ \text { Earth: } & \rho_{\min }=\rho_{\max }-5.514 \cdot 10^{3} \mathrm{kgm}^{-3} & G_{\mu \nu}=-10,29 \cdot 10^{-23} \mathrm{~m}^{-2}\end{array}$

In interstellar space, the density of the space is at maximum and the curvature of space is zero. On the Earth's surface, the density of the space is decreased with a corresponding increasing of curvature of space. The proton has on its surface a smaller density of the space and a larger value of the curvature compared to a neutron star and Earth. The smaller density of the space around a proton compared to a neutron star corresponds with recent research confirming that the peak pressure near the centre of the proton exceeds the peak pressure in neutron stars [7], which both have origin in the density of the space that is smaller in the proton than in the neutron star. The smallest density of the space occurs on the surface of the black hole where the curvature of space is the largest.

\section{The Lorentz factor and variable density of the space}

GPS suggests an abolition of inertial systems in Special Relativity (SR), leading to a search for a more elegant model to better correspond with physical observations. With this improved model, the Lorentz factor $\gamma$ expresses a diminished rate of clocks and a diminished velocity of material changes. The Lorentz factor is defined as

$$
\gamma=\frac{1}{\sqrt{1-\frac{v^{2}}{c^{2}}}}
$$

where $v$ is the relative velocity between the inertial reference frames and $c$ is the speed of light in a space.

In the famous example of a train passing a station, $t^{\prime}$ is the elapsed time on the train and $t$ is the elapsed time at the station, such that

$$
t^{\prime}=\gamma \cdot t
$$

This diminished rate of clocks on the train has its origin in the decreased density of the space inside the train. In general, a moving system interacts with the space energy so that the higher the velocity $v$, the stronger the interaction and more space energy is integrated into the moving object, which in turn increases its mass $m$ according to 


$$
m=\gamma \cdot m_{0}=m_{0}+\frac{E K}{c^{2}}
$$

where $m_{0}$ is the object's rest mass, EK is moving object kinetic energy in the form of integrated energy of space and $\gamma$ is the Lorentz factor.

The moving physical object mass $m$ can be expressed as:

$$
m=\gamma \cdot m_{0}=\left(\rho_{\max }-\rho_{\min R}\right) \cdot V
$$

where $\gamma$ is the Lorentz factor, $m_{0}$ is the rest mass, and $\rho_{\min R}$ is the density of the space at the surface of the moving object. This mass increases because the moving object interacts with the space and absorbs some of its energy which actually is its kinetic energy EK. Equation (7) confirms that because of its speed, $v$, the train's mass increases and the density of the space $\rho_{\min R}$ at the moving train's surface decreases more than for the train at rest. This decreased density of the space $\rho_{\min R}$ causes the rate of the clock on the train to run slower.

According to Equation (7), we can express the Lorentz factor as

$$
\gamma=\frac{\left(\rho_{\max }-\rho_{\min R}\right) \cdot V}{m_{0}}
$$

which confirms it depends on the density of the space on the surface of the moving object $\rho_{\min R}$, the density of the space in interstellar space $\rho_{\max }$, and the volume, $V$, of the object. The minimal density of the space $\rho_{\min R}$ depends on the speed $v$ of the physical object. So, the higher is the speed $v$, the stronger is the interaction of the object with the space, absorption of the space energy is greater, and the density of the space on the surface of the object $\rho_{\text {min }}$ becomes smaller. With a smaller density of the space, the rate of the clock is slower on the surface of the physical object:

increased velocity $\rightarrow$ increased absorption of the space energy $\rightarrow$ decreased density of the space $\rightarrow$ decreased rate of a clock.

For smaller physical objects (compared with stellar objects), such as satellites and trains, a minimal density on their surface is a good approximation for the density of the space inside the object. However, this assumption is not valid for stellar objects where the density of the space increases by Newton's shell theorem when approaching the centre of the stellar object [6]. 
We conclude that the relative rate of clocks and the relative velocity of material changes depend only on the variable density of the space. For example, muon decay when approaching the Earth's surface decreases because their velocity increases [8]. With this increasing velocity, the minimal density of the space on their surface decreases, and the time of decay increases. The duration of this decay does not depend on the selected reference system nor the chosen observer. A muon's relativistic decay is valid for all observers and is determined only by the variable density of the space.

\section{Advances of Special Relativity}

The Lorentz factor is not related to some inertial systems as it depends only on the variable density of the space. From this perspective, the space is the absolute reference system in which elementary particles, physical objects, and stellar objects move. Einstein's idea that there is no absolute motion is again examined here. Because of the abolishment of the notion of an ether at the end of the $19^{\text {th }}$ century, the idea of absolute motion was also abandoned. Introduction of a space as the fundamental arena of the universe allows the reintroduction of absolute motion, which is defined as the motion of a physical object with a space as the absolute reference frame.

Without ether, Einstein introduced the concept of 'relative motion', which means there is no absolute reference system in which any given motion occurs. A moving object's velocity can be measured only in comparison with another object's velocity. In the model presented in this article, the speed of light is constant for all observers because all observers exist in the same space and light is the vibration of the space. Space in the universe is not 'empty', space is the fundamental energy of the universe. Such understanding offers a good example of relative and absolute motions. In physical space which is the absolute frame of reference we distinguish relative and absolute velocity. You imagine you drive a car with a speed of $110 \mathrm{~km}$ per hour. 500 metres in front of you is a car moving with a speed of $120 \mathrm{~km}$ per hour. An accident happens and the car in front of you crashes in the wall along the street. The driver luckily survives but the car is totally destroyed. This simple example proves that the 'relative speed' of $10 \mathrm{~km}$ per hour of the car in front of you was not 'real.' The real velocity of the car is the velocity regarding the highway, which is $120 \mathrm{~km}$ per hour. The same is valid for light speed, whose 'real' velocity is the velocity of the light in the physical space and is valid for all observers.

By assuming the space as the absolute reference frame, we can develop an SR theory without contradictions as those that exist with the current SR in the thought experiment of two-photon clocks. Here, we place two identical photon clocks on a moving train where one is positioned horizontally in the direction of motion, and the other is positioned vertically. According to the idea of "length contraction," the horizontal photon clock will shorten in length and tick faster compared to the vertically oriented clock that will not diminish in length. This scenario leads to a contradiction as SR does not predict that the two clocks in the same inertial system will have different rates. The solution is available through the development of an SR model in a three-dimensional Euclidean space with a Galilean transformation and Selleri formula for the variable rate of clocks with no occurrence of "length contraction" [9]. SR 
equipped with such a formalism also describes successfully all phenomena previously described by classical SR [10].

A second contradiction occurs with the rate of the vertical photon clock on the moving train from the perspective of the observer at the station. The classical interpretation states that for the observer at the station, the vertical photon clock ticks slower because they see the photon in the clock moves in a 'zig-zag' direction [11], as illustrated in Figure 1.

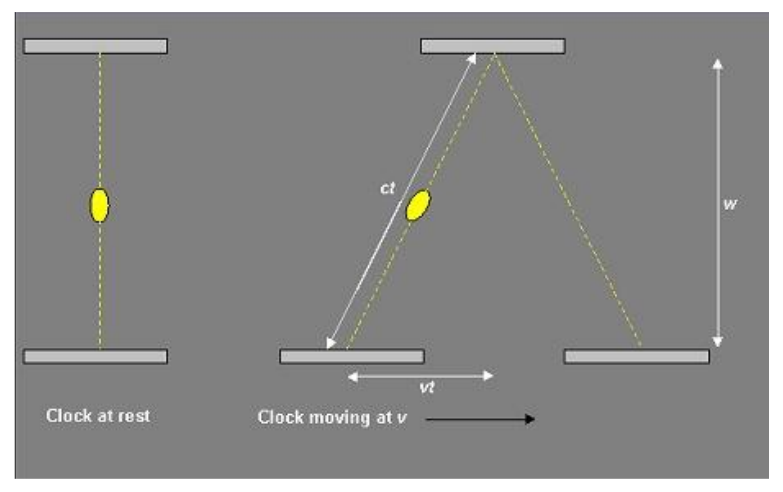

Figure 1. An observer at rest, seeing a moving clock photon.

This explanation may appear illogical because the optical illusion of the stationary observer cannot slow the rate of the moving clock. Instead, in the moving train, the density of the space diminishes, causing a reduced velocity of the photon. Therefore, the moving vertical photon clock ticks slower and its rate is valid for all observers.

\section{Advanced General Relativity}

In the model presented in this paper, the rotation of stellar objects also causes rotation of the surrounding space. For example, the rotation of the space around the Sun causes precession of the planets [12] according to

$$
\sigma=\frac{24 \pi^{3} L^{2}}{T c^{2}\left(1-e^{2}\right)}
$$

where the perihelion shift $\sigma$ is expressed in radians per revolution, $L$ is the semi-major axis, $T$ is the orbital period, $c$ is the speed of light, and $e$ is the orbital eccentricity. The mass of the Sun is not included as there is also no mass of a planet, so these masses do not affect the precession of the planets. In the model of DVR, the perihelion shift $\sigma$ depends on the rotation of the space caused by the rotation of the Sun, which in turn pushing the planets and causes a perihelion precession. With increasing distance from the Sun, the impact of the rotating space on planets diminishes along with the precession of the perihelion. 
Irregular and spiral galaxies comprise approximately $60 \%$ of all galaxies in the universe. In the centre of most spiral galaxies exist a rotating black hole [13], which also rotates the surrounding space causing the shape of the spiral galaxy. Dragging effects between the rotating black hole and rotating space diminishes with the distance from the black hole leading to the spiral geometry.

In the central black hole, matter is continuously disintegrating back into the energy of the space providing a form of rejuvenation of the universe where "old matter" is transformed back into the energy of the space [6].

"The rotating space around the black hole as the physical origin of the surrounding rotating disc was discovered recently, "as if black holes weren't mysterious enough, astronomers using NASA's Hubble Space Telescope have found an unexpected thin disk of material furiously whirling around a supermassive black hole at the heart of the magnificent spiral galaxy NGC 3147, located 130 million light-years away. The conundrum is that the disk shouldn't be there, based on current astronomical theories" [14]. In current astronomical theories, stellar objects rotate in an "empty space." However, in model presented in this article, stellar objects rotate in the medium of a dynamic space, and this rotation, in turn, rotates the space. Therefore, the rotating space builds the rotating disc of the spiral galaxy NGC 3147.

In GPS, Sagnac effect corrections make the system work [15]. Essentially, a signal when moving from $A$ to $B$ in the direction of Earth's rotation needs less time compared to when moving from $B$ to $A$ in the direction opposing Earth's rotation. In a dynamic space model presented in this article, light has a constant speed regardless of the dynamic space's motion. So, when moving from $A$ to $B$, light needs less time because it is moving in the same direction as the dynamic space.

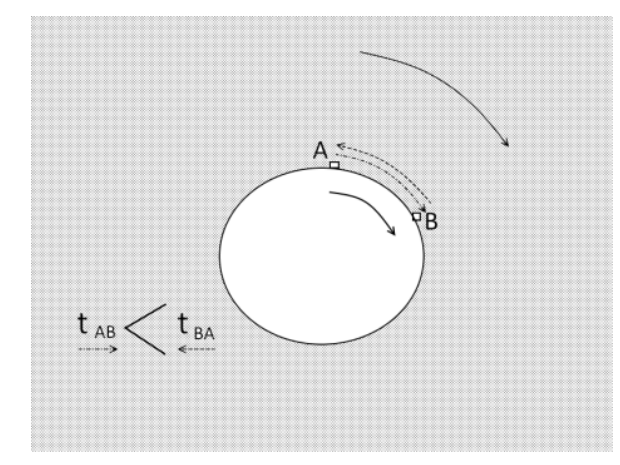

Figure 2. A light signal's time dilation due to the rotation of the dynamic space.

The Michelson-Morley experiment demonstrated a null result because the area of the space around the Earth is not only rotating with the Earth but is also moving with the Earth, as shown in Figure 3. 


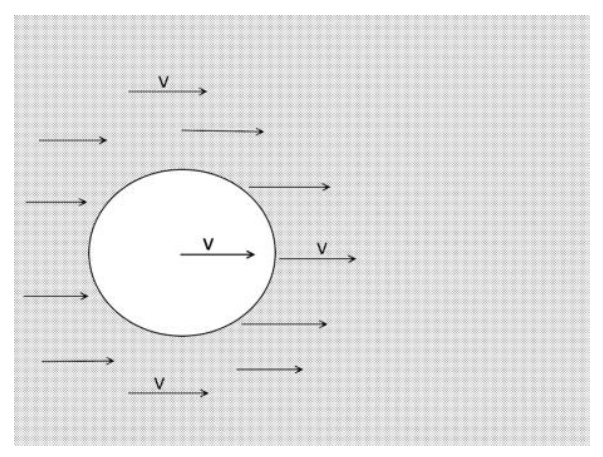

Figure 3. The space moves with the Earth.

So, the negative outcome of Michelson-Morley abolished the ether model. According to bijective research methodology, space is neither filled with ether, nor is it 'empty.' Instead, space contains material objects that contain energy. Energy and matter cannot exist in an 'empty' space deprived of all physical properties, so in this article universal space which is dynamic (it interacts with physical objects) is understood as the primordial energy of the universe. A photon is a wave of the dynamic space, and the velocity of this photon wave is the speed of light, $c$. The photon velocity $c$ is invariant with respect to the space's motion as it appears through the Sagnac effect. The photon velocity diminishes minimally when a photon moves through a stronger gravity where the density of the space is lower, as is the case with the Shapiro experiment, as will be described in Section 5.2.

Motion and rotation of the space with physical objects is referred to as the 'space dragging effect' in this article. This effect was measured by Josef Lense and Hans Thirring in 1918 and was called 'frame-dragging' due to space-time being distorted by rotating objects [16]. Recent research confirms that this 'space-time' model has no physical reality [6], so it cannot be dragged by rotating or moving objects. According to bijective research methodology, an adequate term would be the 'space dragging effect.'

In this article, the idea of "length contraction" and "time dilation" do not exist. Length contraction in SR is only a mathematical tool with no physical reality. By using "length contraction," Einstein achieved a constancy of light in all inertial systems. On the other hand, all observers measure the same value for the velocity of light because the light is the vibration of the medium (dynamic space) in which all observers move. Also, time, being the fourth dimension of space, does not "dilate," and instead, is a result of the velocity of material changes (the rate of clocks included) that depends on the variable density of the space [6].

\subsection{Gravitational redshift}

Gravity has its origin in space fluctuations that interact with photons to diminish their frequency, which is referred to as 'gravitational redshift.' When light from distant galaxies reaches the Earth, its frequency is lower. On its path to Earth, light loses some of its energy because it is moving against the gravity flow of the space that points toward the direction of galaxies, so that 


$$
E_{\text {photonEarth }}=E_{\text {photongalaxy }}-\Delta E
$$

where $E_{\text {photon.galaxy }}$ is the energy of the photon at the galaxy, $E_{\text {photon.Earth }}$ is the energy of the arrived photon at the Earth, and $\Delta E$ is the loss of energy due to the gravitational flow of the space,

$$
\Delta E=h \cdot \Delta v
$$

where $h$ is Planck's constant and $\Delta v$ is the decrease of the photon frequency due to gravitational space fluctuations.

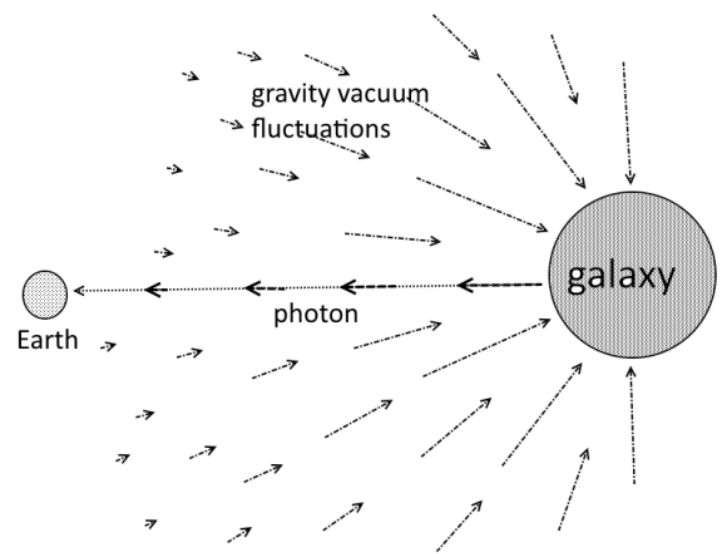

Figure 4. The redshift of light arriving from galaxies caused by space fluctuations.

Because of different densities of the space and corresponding space fluctuations that carry gravity, the frequency of light also changes when moving from the source to the receiver at the Earth's surface. In a Harvard University experiment, a source on the Earth's surface and a receiver at the height of 22,5 meters were positioned, as illustrated in Figure 5.

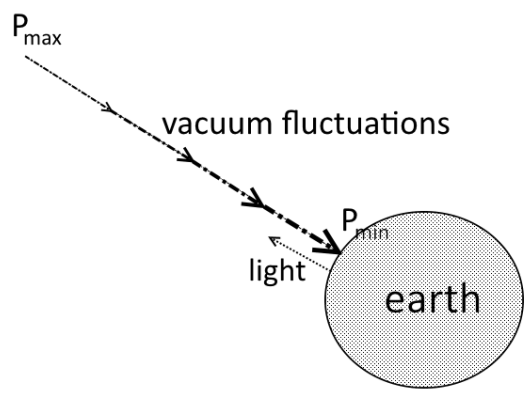

Figure 5. The redshift of light from the Earth's surface. 
The Mössbauer effect was used to measure the difference between y-ray emission and absorption frequencies at each end of the experiment. The measurement accuracy was $\Delta \omega / \omega \approx 10^{-15}$, which shows a change of light frequency as

$$
\frac{\Delta \omega}{\omega}=\frac{G M}{R^{2} c^{2}} \cdot h
$$

where $M$ and $R$ are the mass and radius of the Earth, respectively [17].

We can substitute into Equation (14) for the mass $M$ with the $\left(\rho_{\max }-\rho_{\text {min }}\right) \cdot V$ from Equation (1) as

$$
\frac{\Delta \omega}{\omega}=\frac{G \cdot\left(\rho_{\max }-\rho_{\min }\right) \cdot V}{R^{2} c^{2}} \cdot h
$$

which can be expressed as

$$
\begin{gathered}
\frac{\Delta \omega}{\omega}=\frac{G \cdot\left(\rho_{\max }-\rho_{\min }\right) \cdot 4 \pi R^{3}}{3 R^{2} c^{2}} \cdot h \\
\frac{\Delta \omega}{\omega}=\frac{4 \pi R \cdot G \cdot\left(\rho_{\max }-\rho_{\min }\right)}{3 c^{2}} \cdot h
\end{gathered}
$$

Equation (14) confirms that gravitational redshift depends on the minimal density of the space $\rho_{\text {min }}$ at the Earth's surface, which is the origin of space fluctuations that are fluctuating inward from space towards Earth.

Space fluctuation in the direction from $\rho_{\max }$ towards $\rho_{\min }$ are explaining so called "tired light" model of astronomer Fritz Zwicky. Zwicky proposed that light is losing some of the energy when travelling vast distances from the galaxies to the planet Earth [18].

In model of Relativity here presented space fluctuations from $\rho_{\max }$ towards $\rho_{\min }$ are causing the Pioneer anomaly which means the observed deviation from predicted accelerations of the Pioneer 10 and Pioneer 11 spacecraft after they passed on their trajectories out of the Solar System [19].

\subsection{Shapiro gravitational delay}

In 1964, Shapiro [20] measured the decreased velocity of light in a gravitational field, as observed by the speed of a light signal diminishing when passing the gravitational field of the Sun. Shapiro's result is understood by today's physics as a 'gravitational time delay' caused 
by spacetime dilation, which increases the path length. According to bijective research methodology, this interpretation appears not to be exact as Shapiro did not measure spacetime dilation. In SR, the element of 'spacetime dilation' has no bijective correspondence in the physical world as it has never been observed in physics that spacetime or space are dilating. According to bijective research methodology, Shapiro's result should be termed the 'gravitational diminishing of light-speed' caused by the diminished density of the space. In space with a given gravitational field, the density of the space is diminished that causes a minimal diminishing of the speed of light as defined by the permittivity and permeability of the space

$$
c=\frac{1}{\sqrt{\mu_{0} \varepsilon_{0}}}
$$

where $\mu_{0}$ is the magnetic permeability and $\varepsilon_{0}$ is electric permittivity of the space where there is no influence of gravity, and the density of the space is at its maximum $\rho_{\max }$. In the space with a gravity field, the density of the space decreases and causes minimal diminishing of the permittivity and permeability, which in turn result in the minimal diminishing of the speed of light, as presented by Masanori, "it is known that the speed of light depends on the gravitational potential. In the gravitational fields, the speed of light becomes slow, and time dilation occurs. In this discussion, the permittivity and permeability of free space are assumed to depend on gravity and are variable" [21]. Minimal variability of the speed of light caused by a gravity field maintains SR because its first postulate is valid only in space where gravity is absent. The electric permittivity in flat space with no gravity is $\varepsilon_{0}$, and magnetic permeability in flat with no gravity space is $\mu_{0}$. Following Puthoff, on the surface of stellar object, permittivity and permeability are

$$
\begin{aligned}
& \varepsilon=K \cdot \varepsilon_{0} \\
& \mu=K \cdot \mu_{0}
\end{aligned}
$$

where the space dielectric constant $K$ on the surface of a stellar object is

$$
K \approx 1+\frac{2 G M}{r c^{2}}
$$

with $G$ being the gravitational constant, $M$ the mass, and $r$ the distance from the origin located at the centre of the mass $M$ [17].

For this article, we introduce the heuristic equation 


$$
K=\frac{\mu}{\mu_{0}}=\frac{\varepsilon}{\varepsilon_{0}}=\frac{\rho_{\max }}{\rho_{\min }}
$$

which shows the space dielectric constant depends on the variable density of space. In this sense, a diminished density of the space increases permittivity and permeability of the space which, in turn, minimally decreases the velocity of light as:

$$
c=\frac{1}{\sqrt{\varepsilon \cdot \mu}}
$$

where $\varepsilon$ is the permittivity and $\mu$ is its permeability of the space with gravitational field. From this, it follows that the Shapiro gravitational time dilation has its origin in the diminished density of the space near the stellar objects, which increases the dielectric constant $K$ of the space and minimally decreases the velocity of light. In other words,

diminishing space density $\rightarrow$ increases the dielectric constant $\rightarrow$ increases the electric permittivity of the space $\rightarrow$ increases the magnetic permeability of the space $\rightarrow$ decreases the velocity of light

The classic textbook explanation of the Shapiro experiment is that in stronger gravity, time, as the fourth physical dimension of space, dilates causing light to need more time to reach the point $B$ from point $A$ in a space time that acts as the fundamental arena of the universe. This article shows that through a bijective interpretation of data, where data are not interpreted but read directly, requires an exact explanation where the velocity of light is minimally diminishing in a gravity field due to a diminished density of the space.

Doppler effect proves the second postulate of SR, which states that "the speed of light $c$ is a constant, independent of the relative motion of the source." The observer exists in space, and a photon is the vibration of the same space. When the observer moves toward or away from the source of light, they will experience the Doppler effect. With the understanding that the moving observer and the source both exist in the same space and that light is the vibration of the space, the second SR postulate becomes logical. The observer sees the light with a given frequency coming from the source. When the observer moves away from or closer to the source, the frequency of the light diminishes or increases, respectively. In the Doppler effect, the velocity of light $c$ for the moving observer remains constant because light is a wave of the space in which the observer moves and in which the source of light exists. 


\subsection{Gravitational lens}

Space fluctuations bend light, which we refer to as a 'gravitational lens,' and this bending of light as it passes the Sun is one proof of General Relativity. The space fluctuations near the Sun's surface are strongest and push the photons, causing them to bend, as illustrated in Figure 6.

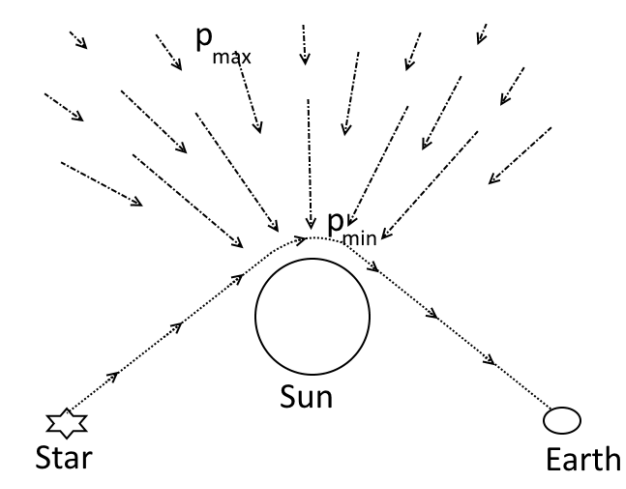

Figure 6. Space fluctuations bending light around the Sun.

Gravity space fluctuations bend the photon's trajectory, which we call 'gravitational lens.' Einstein's formula for the bending of light as it passes the Sun [22] is expressed as:

$$
\delta=\frac{4 G \cdot M_{S}}{c^{2} \cdot b}
$$

where $\delta$ is the angle of deflection, $M_{S}$ is the mass of the Sun, $c$ is the speed of light, and $b$ is the minimum distance between the trajectory and the centre of the Sun. The mass of the Sun, $M s$, can be expressed according to Equation (7), which we can combine with Equations (4) and (20) to obtain

$$
\delta=\frac{4 G \cdot\left(\rho_{\max }-\rho_{\min }\right) \cdot V}{c^{2} \cdot b}
$$

Equation (22) confirms that space fluctuations that carry gravity are directed from space where the density of the space is $\rho_{\max }$, and the density of the space is $\rho_{\min }$ near the surface of the Sun, as in Figure 6. These space fluctuations push the photons, causing light deflection. Bijective research methodology confirms that light passing the Sun is not deflected as a result of the curvature of space. Measurements by NASA have proven that the universe's space has a Euclidean shape [23] so that the curvature of space in the GR model is merely a mathematical description of the variable density of the space. Light is deflected around gravitational objects, such as the Sun, due to a push from space fluctuations. 


\subsection{Structure of the black hole horizon}

In the model presented in this article, on the surface of a black hole, the density of the space is so low that atoms become unstable and disintegrate back into the energy of the space [6]. In current models of the black hole horizon, the density of the space is the missing element. "The recent observation by the Event Horizon Telescope of the shadow of the supermassive black hole M87 has provided the most direct image of a black hole thus far, opening the gate directly observe long-existing and weakly perturbed black holes 42,43 . Our findings indicate that gravitational waves will allow us to dig even deeper and resolve the evolving structure of the horizon itself of newborn and fully dynamical ones" [24]. In this article is considered that a black hole exists within a space with not enough space density at the horizon to maintain the stability of atoms, we obtain a more exact model of the black hole horizon where atoms become unstable and fall apart into elementary particles and further back into the energy of space [6]. The transformation of "matter $\rightarrow$ space energy" occurring at the black hole horizon is the source of gravitational waves.

In this article gravitational waves are not represented as "ripples in the fabric of spacetime" [25] because space-time, as the fundamental arena of the universe, does not exist. Gravitational waves are ripples of the space. Gravitational waves change the permittivity and permeability of space. As gravitational waves enter the LIGO interferometer, they changed permeability and permittivity of the space which minimally changes the speed of light moving in the beams of the interferometer. This minimal change in the speed of light is what is directly measured by LIGO. No direct data exists to confirm that the length of the beams of the interferometer change due to the gravitational waves. How the subtle phenomena of a gravitational wave could shrink or elongate the length of the interferometer beams, which have a solid iron-concrete core, is an unanswered question. The model here presented solves this question through the reading of the available data as required by bijective research methodology. What is measured by LIGO is the minimal change in light speed due to minimal variations of the permittivity and permeability of the space caused by the gravitational wave entering the interferometer.

In the model presented, there exists the similarity between the photon and gravitational wave as both are a wave of the universal space. Recent research confirms gravitational waves have a speed close to the speed of the photon, "since the recent major discovery in physics, the first measurement of gravitational waves [74], achieved by the LIGO/Virgo collaboration, several events have been registered. In particular, the merging of two neutron stars detected with its electromagnetic counterpart by the FERMI satellite [26, 27] has led to implications of paramount importance. One of them is the speed of gravitational waves now constrained to be extremely close to that of light, at the 10-15 level, at low redshifts" [26]. In the model presented in this article the photon and gravitational wave are both waves of the space. 


\section{Technological application of DVR}

The novelty of the model here presented is the variable density of the space. Developing technology to manipulate the density of the space will enable antigravity. Imagine a spaceship on the Earth's surface with an antigravity device that can increase space density. Through this, the spaceship can move in the direction of maximum space density, meaning it will move away from the Earth's surface towards interstellar space. As the density of the space on the Earth's surface is $\rho_{\min }=\rho_{\max }-5.514 \cdot 10^{3} \mathrm{~kg} / \mathrm{m}^{3}$, an antigravity device could locally increase the space density $\rho_{s}$ inside the spaceship. The area of the local increase would move in the direction of maximum space density $\rho_{\max }$ representing motion away from the Earth. The spaceship will stop when its local space density $\rho_{s}$ is equal to the space density outside the spaceship, as in Figure 7.

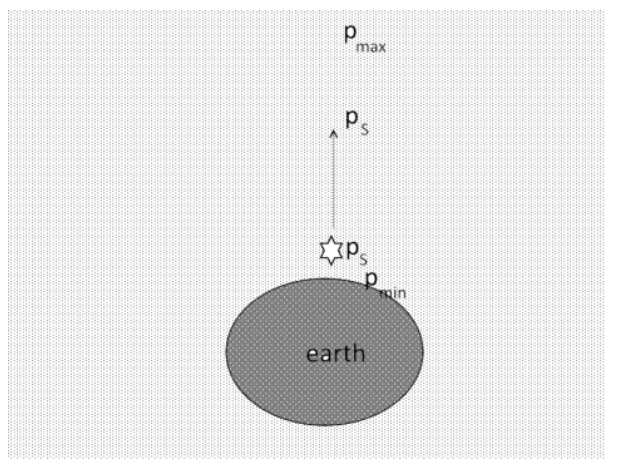

Figure 7. Illustration of using antigravity to leave the Earth's surface.

\section{Summary}

The development of Relativity Theory led to the abolishment of space-time as the fundamental arena of the universe and replaced it with space as the fundamental energy of the universe where time is the duration of motion. Universal space is dynamic in the sense that has a variable density that rotates and moves with physical objects. As light is the vibration of space, the speed of light is invariant from the rotation and movement of the space, and it is minimally diminished in regions where space has lower density. All 'relativistic phenomena' have a physical origin in the variable density of the space and the resulting 'space dragging effect.'

\section{References:}

1. Fiscaletti D, Sorli A. Perspectives of the numerical order of material changes in timeless approaches in physics, Foundations of Physics, 45(2):105-133, (2015).

2. Fiscaletti $D$, Sorli $A$. Searching for an adequate relation between time and entanglement. Quantum Studies: Mathematics and Foundations 2017; 4(4):35774. 
3. Fiscaletti, D., \& Sorli, A. (2018). Quantum Relativity: Variable Energy Density of Quantum Vacuum as the Origin of Mass, Gravity and the Quantum Behaviour. Ukrainian Journal of Physics, 63(7), 623. https://doi.org/10.15407/ujpe63.7.623

4. Neil A., Relativity and GPS, Physics Today (2002).

5. Agnew, D.C., Larson, K.M., Finding the repeat times of the GPS constellation. GPS Solutions. 11 (1): 71-76. (2007).

6. Sorli A. S., Mass-Energy Equivalence Extension onto a Superfluid Quantum Space, Scientific Reports, volume 9, Article number: 11737 (2019)

7. V. D., Elouadrhiri L., Girod F. X., The pressure distribution inside the proton, Nature, 557, 396-399 (2018).

8. Liu L., The Speed and Lifetime of Cosmic Ray Muons, http://web.mit.edu/lululiu/Public/pixx/not-pixx/muons.pdf (2007.

9. Sorli, A., Klinar, D., Fiscaletti, D., New insights into the special theory of relativity, Phys. Essays 24,2 (2011).

10. Fiscaletti D., Sorli A., About a new suggested interpretation of special theory of relativity within a three-dimensional Euclid space. Annales Universitatis Mariae Curie-Sklodowska, sectio AAA-Physica, 68: 39-62. (2015).

11. Philip Harris, Special Relativity, pp. 29 https://web.stanford.edu/ oas/SI/SRGR/notes/srHarris.pdf

12. Einstein, Albert (25 de noviembre de 1915). «Die Feldgleichungun der Gravitation». Sitzungsberichte der Preussischen Akademie der Wissenschaften zu Berlin: 844-847 (1915).

13. Loveday J., The APM Bright Galaxy Catalogue, Monthly Notices of the Royal Astronomical Society. 278 (4): 1025-1048. arXiv:astro-ph/9603040 (1996).

14. NASA, Hubble Uncovers Black Hole Disk that Shouldn't Exist, https://www.nasa.gov/feature/goddard/2019/hubble-uncovers-black-hole-thatshouldnt-exist (2019).

15. Ashby N., The Sagnac Effect in the Global Positioning System. In: Rizzi G., Ruggiero M.L. (eds) Relativity in Rotating Frames. Fundamental Theories of Physics, vol 135. Springer, Dordrecht (2004).

16. Lense, J., Thirring H., "Über den Einfluss der Eigenrotation der Zentralkörper auf die Bewegung der Planeten und Monde nach der Einsteinschen Gravitationstheorie". Physikalische Zeitschrift. 19: 156-163. [On the Influence of the Proper Rotation of Central Bodies on the Motions of Planets and Moons According to Einstein's Theory of Gravitation] (1918).

17. Puthoff H.E., Polarizable-Space(PZ) presentation of general relativity, Found.Phys. 32, 927-943. (2002).

18. Zwicky F., On the Red Shift of Spectral Lines through Interstellar Space. PNAS 15:773-779. (1929).

19. Nieto, M. M.; Turyshev, S. G.,Finding the Origin of the Pioneer Anomaly. Classical and Quantum Gravity. 21(17): 4005-4024. (2004).

20. Shapiro I.I., Fourth Test of General Relativity. Physical Review Letters. 13 (26): 789-791. (1964). 
21. Masanori S., Gravitational effect on the refractive index: A hypothesis that the permittivity, $\varepsilon 0$, and permeability, $\mu 0$ are dragged and modified by the gravity https://arxiv.org/vc/arxiv/papers/0704/0704.1942v3.pdf

22. Albert Einstein, Lens-like action of the star by the derivation of light in the gravitational field, Science, Vol. 84, Issue 2188, pp. 506-507. (1936).

23. NASA https://wmap.gsfc.nasa.gov/universe/uni_shape.html (2014).

24. Juan Calderón Bustillo, Chris Evans, James A. Clark, Grace Kim, Pablo Laguna, Deirdre Shoemaker, Gravitational-wave imaging of black hole horizons: Post-merger chirps from binary black holes, https://arxiv.org/abs/1906.01153 (2019).

25. LIGO, Gravitational Waves: Ripples in the fabric of space-time, https://space.mit.edu/LIGO/more.html (2008).

26. Louis Perenon, Julien Bel, Roy Maartens, Alvaro de la Cruz-Dombriz, Optimising growth of structure constraints on modified gravity, https://arxiv.org/abs/1901.11063 (2019). 